without success, but on the third introduction I drew out the iron from the eye. A drop of solution of atropia was instilled, and both eyes closed with a roller bandage.

On the second day there was marked chemosis but no pain. The wound healed nicely, and the operation looked as if it would prove a success. However, after the third week the eye became soft, $T-2, V=$ nil.

One month after the first operation I enucleated the eye. On apening the eyeball 1 found total detachment of the retina, which stretched in a funnel-shape from the O.D. to its ciliary attachment. At the former site of the le $e_{s}$ was an encapsu lated mass of pus the size of a pea.

Strict antisepsis, so far as was possible in the old R. R. Hospital at Paducah, was attempted in this operation. But the eye was inoculated with pathogenic germs in some way, either through the piece of iron or through the instruments. As the wound in the cornea and iris healed so promptly I am inclined to believe the pathogenic matter was introduced by the foreign body.

Case 2.-J. H. P., aged 26, white, an employe of I. C. R. R., was referred to me by Dr. D. G. Murrell. On June 26, 1898, nine days before I saw him, he was struck in the right eye by a piece of iron, which cut through the cornea and iris (outer quadrant), and pierced the lens at its outer edge, leaving a localized opacity at site of its passage. The pupil was widely dilated with atropia; the eye was not tender on palpation there was no pain; eye but slightly injected: T. quite normal $V=f$. at four feet. The fundus could be seen quite clearly, but I could not make out any sign of the foreign body being in the eye. As these deep wounds in the eye are made sometimes by a sharp corner of a flying particle which does not penetrate the eye, but after wounding it passes on, I beeitated in my diagnosis, thinking it safer to wateh the eye for a time than to draw a too hasty conclusion.

On July 19 vision remained at counting fingers at four feet, but the eye was irritable and slightly sof tened (T-1). On consultation with Dr. Murred, we decided to try the magnet on ithe eye. So on July 21, with the assistance of Drs. Murrell, Taylor and Fort, under chloroform anesthesia, I made an equa torial incision into the eye between the external and lower recti muscles. On the third withdrawal of the magnet point from the vitreous chamber, the iron, which weighed one grain, was brought to the opening, which had to be enlarged to allow it to be extracted. The wound in the selera was elosed by drawing the conjunctiva over it with two sutures. Seven daye later the wound had healed; irritation almost disappeared and the Tn. The fundus was quite elear, except some floating particles in the vitreous. There no detachment of the retina, $\mathrm{V}=\mathrm{i} / \mathrm{z} 0+$.

Fi was on this day, one week after the operation, discharged from the hospital. On August 21, one month after the operation, his vision equaled 15/200. On Oetober 24 I met this patient on the street. His -ye looked as natural, full and bright as the uninjured eye. Patient said the eye was doing nicely. I had no opportunity of testing the vision on this occasion.

The magnet operation is a substitute for enucleation in cases where an eye contains a piece of steel or iron. The object we wish to attain by this operation is to preserve the eyeball. If perchance, we save an eye that retains some, or useful vision, which is not an infrequent sequence, then so much happier is the result of the operation. The magnet (small electromagnet; I have had no experience with Haab's powerful magnet) is not infallible. We can not reason that if, after the magnet point has been introduced into an eye three or four times, in as many different directions, and has not brought out the offending particle, there is none in the eye. The particle may be so entangled, encysted by exudate, or so forcibly driven into the inner walls of the eye that the magnet will not be strong enough to dislodge it. Nor when the foreign budy is removed is the eye free from dungers. Suppuration or chronic irido-cyclitis may follow.

I quote two extracts from Knapp on these points (Arch.Opth., Vol. xiv, p. 307): ". . . Even when the foreign body is removed the disease may progress; suppuration, or, what is more chronic, irido-cyclitis, may develop and threaten the other eye. I therefore make it a rule before $I$ undertake to remove a foreign body from the interior of the eye, to obtain discretionary power from the patient to remove the eyeball whenever I find it advisable. I resort to enucleation without much hesitation when the wound is in the ciliary region, and the extraction of the foreign body offers even moderate difficulty, requiring several introductions of the tip of the magnet, and more probing of the interior in order to find and get hold of the foreign body."

Again, when the foreign body can not be located (Arch.Ophth., Vol. xiv, p. 308): "It seems legitimate, even when the foreign body can neither be seen, nor by an exudation or a limitation in the field of vision be localized, to open the capsule of the eye in the center quadrant, introduce the point of a magnet . . directly to the bottom of the vitreous then witdraw. In case the foreign body is not reached, a second or third attempt, cautiously probing a larger area of the interior of the eye can be made with sufficient safety; but when the foreign body then is not brought out, it is more advisable to enucleate the eye than to make further attempts, because rude dealing with the interior of the eye, even if the foreign body is at last removed, frequently enough causes cyclitis, which is dangerous for both eyes.

A successful issue in these cases is most promising when the foreign body has not passed through the ciliary zone: when it can be located in the eye; when. the eye is seen early; before inflammatory changes take place, or traumatio cataract has precluded examination of the fundus.

This operation should always take precedence of enucleation in these cases, except when the eye has been so torn and lacerated by a large foreign body that any hope of saving it is out of the question, then enucleation should be done at once.

When success follows this operation how beautiful are the results. Instead of the sunken, unsightly orbit, or the staring artificial eye, we preserve the human face in all its natural appearance, and even more, in many instances, preserve an eye retaining a useful amount of vision.

415 Broadway.

\section{CONCEALED TRAUMATIC MENINGEAL HEMORRHAGE.}

Read at the Fifth Annual Meeting of the American Academy of Railway Surgeons, held at Chicago, Oct. 5 to $7,1898$.

BY H. REINEKING, M.D., Surgeon C. \& N. W. R'y. SHEBOYGAN, WIS.

The appropriateness of the title of my paper may, strictly speaking, well be questioned, for to the wellinformed physician or surgeon no intracranial hemorrhage, of any considerable amount, could long remain concealed. I have applied the term, and shall limit my remarks, to cases in which there is absence of outward flow of blood and of fracture or fissure of the cranium, but in which the presence of a hemorrhage is usually indicated by unmistakable symptoms relating to the intellectual, sensory and motor functions. The following case illustrates so typically the usual symptomatology and course, as well as the results to be expected both under the expectant and the operative plans of treatment of this important class of cases, that I wish to put it on record, and might woll offer it in lieu of any dissertation on the subject. It, 
in attempting to present briefly the most advanced views on this subject, so important to the railway surgeon, I shall succeed in calling forth discussion and eliciting an expression of the experience of the Fellows present, I shall have reached my purpose.

Case 1.-C. W., a farmer, aged 54 years, of good previous health and good habits, was injured May 13, 1898, in a runaway. So far as could be ascertained, he was thrown from his wagon and struck his head on the stone curbing. He was stunned for a few minutes, then regained semi-consciousness and never afterward became fully unconscious. According to the statements of the attending physician, he acted, during the first few hours after injury, very much like a person under the excitement of alcoholic intoxication-wanted to go home, and was very unmanageable. Up to the time of being put to bed, which was nearly an hour after the injury, he seemed to have full control of his extremities. Aside from a few bruises on various parts of the body, there were two deep scalp wounds in the upper portion of the right parietal region, but no evidence of fracture or fissure of the skull was found at this or any subsequent time. The wounds were dreseed and the patient put to bed and quieted by a hypodermic injection of mor. phia. The next morning the left side of the face, trunk and upper and lower extremities were found paralyzed, both as to sensation and motion; delirium continued, temperature and pulse were normal. The exact time at which the hemiplegia set in could not be determined.

On my first examination, two days after the injury, there was complete left hemiplegia ; the pupila equal, fixed between dilatation and contraction; both eyeballa turned upward and to the right, the patient able to turn them to the left to a slight degree, but unable to hold them under control beyond a few seconds. The tongue was protruded normally. The patient was partly comatose, at other times very restless, talking incoherently. Urine and feces were mostly passed unconsciously. Temperature and pulse were slightly above normal, breathing not disturbed. The symptoms described pointed unmistakably to the existence of a clot, producing pressure over the motor area of the right cerebral hemisphere, and of sufficient size to interfere with the function of this entire area. The scalp wounds were found infected; they were, therefore, reopened, enlarged, and treated openly. A very thorough examination of the skull made at this time failed to show the least injury to the bone.

The conditions described called urgently for operative relief of the pressure, and this was promptly advised, but it was not until May 21, eight days after the injury, that full consent was obtained and the operation undertaken. Meanwhile there had not been the slightest improvement in the symptoms, showing that little was to be expected from absorption or contraction of the clot. Hemiplegia was absolute; urine and feces were passed automatically; mental condition was very irrational, with great restlessness.

In planning the operation and deciding as to the point at which I should enter the cranium, I took into consideration the length of time elapsed since the injury, which, together with the stationary character of the symptoms, made it almost certain that there would be no necessity of ligating any vessel at this time: it was also evident that an opening made anywhere over the motor area of the injured side would be certain to expose the clot. The location of the scalp wound was there fore decided apon, the wound slightly enlarged downward, and the trephine applied at a point four and one-half inches behind the external angular process of the frontal bone, and four inches above the external auditory meatus. This locality proved a favorable one for the evacuation of the clots, but subsequent events showed that for the purpose of good drainage, an opening farther back would have been even more advan tageous.

The opening was made with a three quarter-inch trephine. As soon as the button of bone was lifted, the black mass of clotted blood presented itself, showing it to be extradural, While the mass showed slight pulsation, imparted to it from underlying vessels, it did not bulge into the opening, owing probably to the length of time the pressure had existed and to the consequent loss of elasticity in the compressed structures. The clots were turned out with a small Volkmann spoon and a strong current of sterilized water from a fountain eyringe. They were found to cover an area measuring abou five inches antero-posteriorly and two and one-half inches vertically, and separating dura and cranium for fully an inch at the central portion of the mass. As the greater portion of this space lay below and behind the opening, the latter was en larged in these directions with a rongeur forceps, until free access was had to every part of the cavity. Hemorrhage was slight; there was no evidence of laceration of the dura, no roughness of the inner table. It soon became apparent that there was not the slightest tendency for the brain to expand and fill the cavity, so the latter was loosely packed with iodoform gauze, the wound left entirely open, and a gauze dressing applied. After the operation the temperature, which on the day previous had been 102.2 , fell at once and, after four days, was normal and remained so. Pulse, after operation 124, came down to 90 in a few hours and remained good.

The patient's mental condition began to improve almost immediately, and always kept in advance of the improvement in the functions of motion and sensation. Involuntary passage of urine and feces ceased after three days. An early sign of improvement was the return of normal action of the ocular muscles and of the pupils. Then the face resumed a natura expression, together with the return of sensation and motion on the affected side. For two days after the operation there was not the least evidence of improvement in the extremities. On the third day the patient could move the hand and fingers, on the fourth could raise hand and forearm. On the fifth day motion wae first noticed in the foot, and he could raise his hand to the face. Motion in the toes returned on the ninth day, on the eleventh he lifted leg and foot slightly, and could move them freely the next day. Sensation returned slightly in advance of motion.

The intellect had by this time become quite clear, and his general condition was good. But the local conditions were not so satisfactory; the cavity had diminished but slightly, and there was a large amount of discharge of a reddish-brown liquid which was but imperfectly removed by the gauze drain. A small dressing forceps could still be passed about freely in the cavity, and could be passed backward and downward for a distance of four inches. A counter-opening was accordingly made at a point four inches behind and below the first opening and the same distance from the external auditory canal, which brought it over the most dependent portion of the cavity when the patient was resting on his back. A gauze drain was inserted from opening to opening, and further packing dispensed with. This afforded good drainage, the cavity diminished rapidly, and healing was soon complete. Meanwhile the improvement in regard to intellect, sensation and motion had been rapid. June 9, nineteen days after the first operation, he walked about his room unaided, and soon regained the full control of his extremities. He left the hospital June 24, just six weeks from the day of injury. August 31, nearly four months after the injury, he called at my office, to all appearances a perfectly well man. The trephine openings had become filled with hard cicatricial masses, apparently containing con siderable bony callus, and no tendency to hernia cerebri could be detected.

Of the clinical features of the case, the absence of dilatation of the pupil of the injured side was unusual, considering the great increase of intracranial pressure and the regularity with which it is found in similar injuries. As the modus operandi of the effects of intracranial pressure on the pupil is not understood, I will venture no theory for the explanation of the absence of the dilatation in this case.

The important questions which confront the surgeon in the consideration of this class of cases are: 1. Whether to operate; and if so, at what time? 2. The choice of the most advantageous point for opening the skull. 3. The method of operating, including the choice of instruments for perforating the bone.

As to the first question, whether to operate in a given case, and as to the best time for such interference, it may be said, that, with the indications and diagnosis reasonably clear and certain, the less time is lost in expectant treatment, the better will be the patient's chances, both as to immediate and remote results, provided that he is not in a condition forbidding all operative interference, or that, on the other hand, rapid improvement has not already set in. Not only will the immediate danger of fatal hemorrhage be thus removed, but the deleterious and often permanent effects of long-continued pressure will also be avoided. But on the other hand, the surgeon should not hesitate to undertake the operation, even if called at a late stage of the case, as very good results are 
known to have followed on surgical measures instituted many days after the injury.

The diagnosis is, as a rule, not difficult. The symp. toms will vary in minor respects, according to the location of the hemorrhage and the centers pressed upon, and must be interpreted in the light of anatomic and physiologic data and the teachings of cerebral localization. The lapse of a varying interval of time between the receipt of the injury and the onset of unconsciousness, or of hemiplegic symptoms, is one of the most pathognomonic evidences of meningeal hemorrhage, and generally indicates the extradural form. The length or this interval is in inverse proportion to the rapidity of the hemorrhage; it may be entirely wanting where the hemorrhage is rapid, or other grave lesions present, or may be unnoticeable or obscured by unconsciousness due to concussion or other conditions. When present, it serves to distinguish the attack from an ordinary apoplectic seizure. The longer this interval, the more likely is the hemorrhage to be found extradural, as the attachment of the dura to the inner surface of the skull offers some resistance to the accumulation of blood, and thus retards the onset of pressure symptoms.

Inequality of the pupils, due to the dilatation of the one on the injured side, is one of the most constant symptoms, yet too much importance must not be attached to its absence, as shown in the case just reported. The same is true of the deviation of the eyeballs upward and toward the affected side. The condition of the sensorium also varies widely: from perfect clearness of intellect, through all the shades of mild disturbance, to restless delirium and deep coma. In severe forms, the respiration is usually slow, irregular and stertorous, but may remain unaffected, even when other symptoms are very pronounced. Slowness of the pulse is another symptom of some positive value, yet the pulse may be found normal, or even increased in frequency. Hemiplegia of the arm, arm and leg, or of the entire side, generally appears in the order mentioned, and rarely affects the leg alone or in advance of the arm; as a rule it is wellmarked, but is found in all grades of severity. Sufficient diagnostic data can usually be gathered, that, when taken in connection with a history of trauma and evidences of injury to the scalp or cranium, they will suffice to lead the medical attendant to a pretty clear conclugion, both as to the presence of a hemorrhage and to its situation in relation to the brain centers and to the cranium. Cases in which the symptoms are so obscure as to afford no clue as to the location of the clot are not favorable for operation, but whenever the indications are reasonably clear, the patient should be given the benefit of possible relief by surgical interference, as the opening of the skull and dura, if properly done, exposes the patient to but little risk.

As to the second question, the most favorable point for entering the skull, the surgeon must be guided by anatomic considerations, the stage of the case at which he is called, the focal symptoms present, the location of wounds of the scalp, and the requirements for drainage. In all recent cases, unless the focal symptoms point clearly to other localities as the seat of pressure, it will be advisable to make the opening at the point usually recommended in the text-books, viz., one and one-half inches above the zygomatic arch and the same distance behind the external angular process of the frontal bone. Here an opening of an inch in diameter will expose the anterior branch of the middle meningeal artery, which, in the great majority of cases, is found to be the bleeding vessel. If the dura has been separated from the bone, the artery will be found imbedded in this membrane, lifted away from its groove in the bone. If bleeding, it should be ligated with a catgut ligature passed around it by means of a small curved needle, care being taken to include as little as possible of the dural tissue. In case the artery can not be secured, packing with iodoform gauze should be resorted to. The opening is to be enlarged by means of rongeur forceps, as the necessities of the case may require. If the operation is undertaken after the lapse of several days and the pressure symptoms have become stationary; in short, when the necessity for ligating the vessel has pretty certainly passed, the surgeon will first of all be guided by the focal symptoms, and when the compressed area is large, by the other considerations above mentioned.

As to the method of operation, no strict rules can be laid down, except that it must be done under the most rigorous antiseptic precautions, with the least possible amount of injury to the structures protecting the brain. The hemorrhage must be controlled, the clots thoroughly removed, drainage whenever there is a cavity must be thorough, and the results which often remotely follow these traumas must, as far as possible, be guarded against.

There is some diversity of opinion as to the advantages and disadvantages of the trephine on the one hand, and the chisel and mallet on the other, as implements for opening the cranium. It probably matters little which instrument is used, provided the operator is thoroughly familiar with the one he uses. Personally, I consider the trephine best adapted to these cases. The dura is either found separated from the bone, so as to be protected from injury by the instrument, or, if the hemorrhage is intradural, it generally requires free incision, unless lacerated beforehand. The chief argument advanced against the use of the trephine-that of injuring the dura-has no weight, in these cases at least.

Moses Gunn, in an address before the American Surgical Association, in 1882, said: "In this connec. tion I venture to put in a plea for the much-dreaded trephine. The operation of removing a disk from the cranial walls, performed with proper care and skill, is not an 'operation fraught with danger.' There is very little risk of injuring the dura mater with that instrument. If that membrane has not been wounded in the occurrence of the accident for which we resort to the use of the trephine, its safety from injury by that instrument may be pretty confidently assured. And while I thus defend the proper use of the trephine, I desire also to protest against the use of the chisel or gouge on the cranial walls, if the propelling force is applied by a series of blows from mallet. A single blow of the force usually required may be insignificant, bnt the result of a number of them in rapid succession must be proportionate to the aggregate amount of all the blows. Thus estimated, we may not be surprised at symptoms of concussion fol lowing its use." With the use of the rawhide mallets and improved cutting instruments, there is no doubt but that the chisel can be used with perfect safety if the mallet is used with that care and delicacy which should characterize all operative work on the cranium, while in large osteoplastic operations these instruments are simply indispensable. 
Wounds of the dura mater should, when practicable, be sutured, but when the injury is such that this can not be done, a piece of gold foil, large enough to extend beyond the margin of the defects, should be inserted between lura and cortex cerebri. This will prevent adhesions and protect the patient against many of the remote effects of head injury. This gold foil can be prepared by annealing a number of layers of dentist's foil, may be cut into sections to facilitate introduction, and should be stiff enough only to be easily inserted. In the absence of a cavity it will be held in place by mere contact with the tissues. It should be one of the articles constantly in the surgeon's armamentarium. In recent and perfectly aseptic cases, where drainage is not required, the bone removed should be cut into chips, replaced, and the wound closed, or a trap-door-shaped flap of bone and overlying soft structures may be raised, to be put back into place after stopping the hemorrhage and cleaning away the clots. When drainage is required, it is advisable to leave the defect in the bone open, as infection and retention of discharges must above all things be guarded against, danger from this source being far more likely to occur than any subsequent trouble from hernia cerebri.

\section{DISCUSSION,}

Dr. W. W. Grant of Denver-There are several points in this paper worthy of thorough discuesion. In the firet place I believe that the opening in trephining, whether for depression of the skull or not, should be as small as possible and permit the accomplishment of our purpose in operating. Not infrequently the ulterior consequence of an operation-owing to the large cranial defect-has been worse than the disease we have attempted to remedy. Epilepsy quite constantly follows firm cicatricial adhesions between the dura and the cortex, and for this reason many surgeons now hesitate, unless there are de. cided symptoms of compression, to operate, guided as well by the fact that many of these patients do exceedingly well and never have any bad after consequences. I have had personal experience with a number of such cases. To prevent the formation of cicatricial tisene or adhesions, it is a question in surgery what is the best material to use, but gold foil is generally so accepted. Dr. Freeman of Denver, has been experimenting with the lining membrane of egg shell for this purpose, and he has had some satisfactory results which he reports in a recent issue of the Annals of Surgery. Small fractures can be better treated by chisel and mallet than by the trephine, for one may of ten put an elevator under the depressed portion and raise it without removing it. In this way you will minimize the danger and unpleasant consequenzes which result from adhesion of the tissue.

Dr. W. L. Smith of Streator, Ill.-A few years ago I saw an operation on the head. The Doctor quotes the late Dr. Moses Gunn, who was a good surgeon in his day. I have also heard Dr. Gunn say that pus was dead or dying blastema. This statement was ali right for that period. So far as making a hole in the skull where an injury is concerned, if there is not a hole already made, I believe the better plan is to use a chisel and mallet, so that we may have plenty of chance for drainage. We can not secure effective drainage through a three quarterinch trephine hole; neither can we tie an artery if it is bleeding. So far as making pressure to control hemorrhage is con. cerned, I have little faith in it. I have made several operations on the head with chisel and mallet, and have not had any serious results from concussion or anything of that kind, and I be lieve, if we are as careful in making a hole in the head as we are in the abdomen we will have just as good results. If the Doctor had used chisel and mallet and raised the flap of bone, he would have had plenty of room for drainage, and, I think, would not have had to make a second operation.

Dr. Fred J. Hodges of Ashland, Wis. - From an analysis of the case and history related one would interpret gradual hemorrhage from a small vessel. That being the case, I do not think that the inequality of the pupils is a matter of any new or added interest, for the reason that the brain is very tolerant of pressure, when gradually applied, and if this same degree of pressure had been applied as a result of the hemorrhage from a large vessel, there would undoubtedly have then been a difference in the pupils, and the same difference in effect would have been observed in regard to the mental faculties. The mental faculties would have been entirely and quickly extinguished; whereas the pressure being so gradually applied, they were impaired rather than extinguished. I have seen the same group of symptoms produced from an extradural rupture of the superior longitudinal sinus. So even with this group of symptoms it is not safe to assume that you are going to come down on a mass of clotted blood. In a case I operated a short time ago, after making a small trephine opening in the skull, there escaped a column of venous blood at least two and a half inches in height. We waited a few seconds to see if there was a blood clot, but there was no change in the extent or force of the stream. The opening was closed, and another made farther down on the parietal with the hope of getting outside the hematoma. But that giving us the same result, a small piece of gauze was introduced from without to encourage clotting, and the scalp wounds closed. As soon as a blood clot formed the symptoms disappeared, and the patient recovered without any further interference. Bearing this in mind, it is just as well to investigate, as far as you can, the state of affairs before removing the entire button, if you use a trephine, or as soon as you make a small opening, if you use a chisel. I do not know how the case could have been treated if a large button had been fully liberated. Evidently there was a large rent in the sinus and to have controlled that through a large opening would have required considerable ingenuity.

Dr. ARthur D. Bevan of Chicago-I just want to defend Moses Gunn in his definition of pus. I do not believe that any more rational short description of pus has ever been given than that by Dr. Gunn, namely : "Pus is dead or dying blastema." It certainly fills the bill even in our advanced stage of pathologic knowledge. Pns is derived from the white blood corpuscles and the embryonal cells, and although Gunn's knowledge of the more refined pathology involved in the production of pus was not up to our present data, yet I should like to defend the general proposition. In the same way, I should like to defend the use of the trephine in just such cases as the Doctor has described in his paper. I would use the trephine in preference to the chisel, because, as a rule, in cases of rupture of the middle meningeal artery we can locate approximately the point of rupture. I do not say that is constantly so, but the general anatomic rule given is fairly accurate-an inch and a half above the zygoma and an inch and a half behind the external process. A 11\%-inch trephine will locate the seat of rupture in nine cases out of ten, and 90 per cent. of the cases of hemorrhage are from the middle meningeal artery. The trephine exposes a small area, and you can locate it definitely. It is covered by the temporal muscle and deep fascia, and is in a position where the loss of half an inch of bone is not as apt to result in symptoms at the end of a month or years as it would be in the upper portion of the parietal eminence, for instance, where it is not covered by the deep fascia and temporal muscle. With reference to the inability to ligate a vessel through a trephine opening, I should say that I bave done this twice, having ligated the middle meningeal artery through a half-inch wound, and in another instance through a three quarter-inch opening.

I am opposed to the use of gold foil. In a case where we have to remove a small area of bone, especially where it is deeply covered by muscle and fascia, I am inclined to believe that we would be less likely to have epilepsy follow in the lapse of months or years where we had allowed the external periosteum to remain, than where any foreign body is interposed. The use of celluloid, of a gold plate, of gutta-percha tissue, has been tried in a few cases, and the results have been unsuccessful. I know of several cases where either celluloid or gold foil has been employed and it was necessary to remove it. I do not think those cases have found their way into the literature; it is the successful cases that are reported. While not wishing to consume too much of the time of the Academy, I desire to relate briefly a case which I have had recently, illustrating this class of lesions

A man, a bricklayer, was in a shaft; his companion had been working with him. The companion left to go to some other part of the building, was gone about half an hour, and when he returned the man was lying unconscious at the bot tom of the shaft. An ambulance was called; the man was brought to the Presbyterian Hospital; we examined him care fully and found no evidence of any lesion with the exception of a slight contusion on the side of the head. The urine showed sugar; a diagnosis of diabetes was made. We watched the case carefully, as one of probable diabetic coma. The patient died. Postmortem examination revealed rupture of the middle meningeal artery as the cause of the coma and death. I am inclined to think in such cases as that we would be jus tified in making an exploration for diagnosis. I am rather 
inclined to believe from my experience with a few similar cases, that an early operation would have saved the man's life.

Dr. EDward Dooley of Buffalo, N. Y.--I was very much interested in the paper, and from the remarks made by several gentlemen I noticed a great deal of stress was laid on the dan ger of the adhesions that are apt to form. I recall a case that came under my observation about five years ago:

A young man riding on an engine, in jumping off, stı uck the top of the switch frame and sustained an extensive compound, comminuted fracture on the left side of the skull. Much of the bone was broken into small splinters, which I removed without using either chisel or trephine. 1 should say I removed al together an area fully two and a half inches square, including the left parietal eminence and the bone below and in front of it. The dura was lacerated, a blood-clot that bad formed was re moved, the wound united with catgut and dressed with iodoform gauze. The condition of the patient was such that I was in a hurry to get him off of the table, thinking he was going to die, but to my great astonishment the young man recovered. He ouffered from aphasia for ten days, but gradually his intel ligence and speech returned, so that at the end of six weeks he was able to leave the hospital. The young man has been at his occupation as a switchman ever since, it now being five years since he sustained the injury. There is certainly cica. tricial tissue covering the entire space over the left parietal eminence, and he has not had any epileptic seizure or paralysis.

This is an exceptional case. I do not wish to be understood as saying that it is unnecessary to use gold foil, but there are undoubtedly cases where we will not have epilepsy or paral ysis, even though there be a cicatricial scar over the brain substance.

Dr. R. Harvey Reed of Rock Springs, Wyo.--There is one point I would like to refer to in regard to the Doctor's paper. and that is with reference to the instrument which is to be used in opening the skull. I have used the chisel very freely, the trephine and the DeVilbiss instrument, and $I$ do not think that, from a surgical standpoint, it makes much difference which is used. There is an important point in the matter, viewed from a medicolegal standpoint which a personal experience of my own well illustrates.

I was once called to see a man who had been assaulted and left for dead by his assailants. He had been attended by some physicians of the neighborhood until he developed a paralysis of the right side of the face and the left arm and leg some four days later, when I was called in consultation. I recommended operation, which was agreed to. I removed a good-sized flap by means of the chisel and mallet, and over the arm and leg area found the dura bulging out boldly and without pulsation. This naturally led to the expectation of finding a clot under it, but an incision only released a little serum. Aspiration of the lateral ventricles removed about two ounces of blood and serum and allowed the brain to resume its normal relations. Wounds of the dura and scalp healed primarily, and the patient did well for a time, but two weeks later began to do badly and finally died. At the trial of his assailants, the defense assumed the position that the doctor had killed the man. In his argument the attorney said : "Gentlemen of the jury, think of this man taking a great chisel and an enormous mallet and pounding and pounding until he cut a hole in the poor man's skull three inches in diameter. Is not that enough to kill any man?" Of course the murderers were set free and I was discountenanced. Gentlemen, we are treating patients that are corporation cases. and while I feel and know that I contributed in no way to the death of this man by the use of the chisel, yet at the same time if I had used the DeVilbise rongeur, I would have avoided the charge by the attorney of having "pounded his head with a mallet" and would have accomplished the same results. For this reason $I$ believe that it is well to avoid the appearance of evil - at least in such cases as may eventually come before the courts-and by so doing avert trouble for the corporations for which we are working, or as in this instance, turn loose criminals whose place is in the peritentiary.

Dr. W. J. MAYo of Rochester, Minn. - I am going to ask the essayist one or two questions, which I hope he will answer in his closing remarks. The first one is this: In case the focal motor symptoms do not agree with the injury, would he go down on the focal lesion, or go on the side of the injury? I consider this an important point. In two of the three cases which I have seen, the external wound and the motor symptoms did not agree. There is no class of cases more confusing to us than these. A man sustains an injury of this kind and develops thrombosis; you cut down, open up the calvarium, and find an edematous brain, the symptoms coming on soon after the injury. I recall one case, the history of which was exactly that of the case described by the Doctor, yet it was due to a progressive thrombosis. Dr. Reineking is certainly to be congratulated on the result obtained in his case, and it is an encouragement for us to operate whenever we epcounter these cases.

A good deal has been said in regard to the use of the chisel and trephine. I do not think it makes much difference which we use. My brother and myself do considerable surgical work on chronic cases of this character in two large asylums, and I should say that it is a question of choice between the chisel and trephine. I do not think the little taps we give with the chisel would make much difference. I am inclined to think that those who oppose the chisel so strongly have not used it many times. The DeVilbiss instrument is certainly a fine one to work with.

Dr. Reineking. - In reply to Dr. Mayo's question as to where I would trephine in a case where the symptoms disagreed, the injury being on one side and the focal or preasure symptoms on the other, I should first trephine on the side toward which the pressure symptoms pointed, and if unsuccessful there and the symptoms persisted, I should not hesitate to trephine the other side also.

Dr. Fred J. Hodges of Ashland, Wis.-I would like permission to make one further remark in regard to this class of cases. Dr. Mayo touched upon one of the most important points in the whole matter when he asked the question as to the site of the operation. In the July number of the American Journal of the Medical Sciences, for 1894, I think, Walton, one of the senior surgeons of the Massachusetts General Hospital, in an elaborate paper, cites a number cases in which he reaches the same conclusion or lays down the same proposition as that an nounced by the essayist, namely, that he would operate at the site of the focal symptoms. I believe this to be radically wrong. Surgeons have gone far in advance of the physiolo. gists. If you will consult a standard text-book on physiology, you will be reminded that the decussation in the anterior pyr amids varies all the way from no decussation whatever to com plete decussation. It takes a vivid imagination to make a great many reported cases conform with the generally accepted idea among surgeons that injury on one side produces paralysis on the opposite side. If you will study cases in the light of physiology, you will find that decussation is not constant by any manner of means, and the surgeon is misled in operating at the site of focal symptoms. I had a case at the time of this report which beautifully illustrated that point. The man was operated upon for epilepsy about a week after his first convulsive seizure. On examination, there was a scar found on one side of the head which be said marked the infliction of a bullet wound eleven years previously. This was on the right side of the head, and the post-epileptic paralysis was on the right side of the body. Following the Walton rule an operation would have been undertaken on the left side; but following the com mon-sense rule, operation was made on the right side, when two thirds of a 38-caliber bullet was found encysted in scar tissue just within the surface of the cortex. The bullet was re moved, and the scar tissue excised. It is now nearly four years since the operation was done, and the man is absolutely free from any central disturbance.

If you will gather carefully the clinical reports of skull injuries, you will find that it requires a vivid imagination to make the clinical history correspond with the idea of invariable decuesation, and for the best of reasons - it does not exist.

While an interne in Cook County Hospital in this city, a man was brought in with fairly well-marked focal neurologic symp toms following an injury to the head - an injury that was not easily localized externally. A surgeon of this city, who has given a good deal of attention to brain surgery, cut down upon the supposed point of pressure and found nothing. He need led the brain in various directions with the same results. We had the gloomy satisfaction the following day of discovering a large clot on the opposite side of the head. The clot was on the same side as the paralysis. The proper explanation oc curred to none of us at that time. Now I am certain that it was a case in which there was no decussation. Surgeons can not safely go in advance of the physiologists. The site of the injury is a safer, more rational guide to operation than the localization symptoms.

Dr. K. HARvey ReEd of Rock Springs, Wyo,-The remarks of Dr. Hodges recall a case which to me was a very odd and interesting one, and which I will report briefly

A miner was struck on the top of the head by a falling rock, which inflicted an extensive compound comminuted fracture of the skull. I did not think he would live, but felt it my duty to go ahead and do the best I could, so I removed an area of bone, perhaps $2^{1} \%$ x 3 inches, that was completely comminuted. The dura mater was lacerated apparently by almost every piece of bone, and in addition the brain substance itself was crushed. I should say that I removed from that patient 
brain substance to the quantity of at least half an ordinary teacup, and it oozed out for some little time after operation. Being an infected wound, I packed it and allowed it to heal by gramulation. 'To my utter eurprise, on the fourth day after the operation, the man got up and went out of the hospital without a single symptom of paralysis, notwithstanding that the injury was immediately over the leg and arm area. On that day he walked four miles in looking up witnesses for a lawsuit that was to come off the next day. I supposed that if he did not die from the injury he surely would from the effects of so great an exertion. However, he remained in the hospital about two weeks, the wound healed rapidly, and he got well without any paralysis whatever. If we had any fear whatever of the formation of cicatricial tissue, we certainly would have it in a case of that kind. After leaving the hospital he resumed work for a considerable time, and was afterward run over by the cars, the leg and arm taken off, and the last I heard of him he was still living.

Dr. Reineking (closing the discussion)-I purposely did not speak of depression or depressed fracture as indications for operative interference. I discussed cases presenting evidence of intracranial hemorrhage. Personally, I do not recommend the use of gold foil unless the dura mater is lacerated so that it is not readily sutured. I had a very happy experience with the use of gold foil between the dura and cerebral cortex, in a case of epilepsy. The man had as many as three attacks a day, and as many as fifteen or sixteen during the year, and since relieving the adhesions which bound down the cerebral cortex to its coveringe and protecting him against further ad. hesions by the use of gold foil, thick enough to prevent adhesions, the seizures have cumpletely ceased. It is now (October, 1898) eighteen months, since which time he has had no attack whatever. Shortly after the operation he had two slight attacks. I am afraid of adhesions between the dura and cerebral cortex. If we investigate the literature of operations for epilepsy, we will find it occurs where the adhesions and cicatricial tissue were beneath the dura.

In such a case as the gentleman from Buffalo (Dr. Dooley) cited, there was no occasion for using gold foil. I am not surprised that his case did well. We have all seen similar cases. It makes little difference how large the defect in the bone is as long as the adhesions are not deep. Even where they do exist epilepsy will not invariably occur any more than will nerve injuries be invariably followed by a neurosis or paralysis. In the case in which I placed gold foil between the membranes, a skiagraph was taken about a year and a half later, and it showed the plates in position. The defect was large, and the foil, which was flexible, was inserted in four sections. The man has brightened up mentally, has been relieved of epilepsy, and certainly the case is one to make a surgeon a little enthusiastic in the use of gold foil. I am free to confess that I am an enthusiast on this subject. In cases where there is fracture or depreseion, I would use chisel and mallet, a gouge, or a DeVilbiss forceps, but in cases of simple hemorrhage without depreseion or fracture, $I$ think a trephine is the nicest instrument. With it we remove no more than is absolutely necessary; we can enlarge the opening and do it well with a rongeur forceps. The size of the opening is no argument, and it was advised in my paper to enlarge the opening in accordance with the indications. In the case I reported, the bad drainage was not due to the use of the trephine, but to the location of the opening. If it had been made at the most dependent portion of the cavity, the drainage would have been good with the trephine opening, and with a single one. That was the sole occasion for making a second opening.

As I have previously remarked, many of these severe injuries will recover without the use of gold foil; but we never know which are going to be the bad cases any more than we know about injuries to the nerve trunks, etc., and we had better adopt the precaution of using gold foil. Sufficient clinical experience is on record, as evidenced by the cases of Roswell Park, Estes, and othere, that the gold foil can be used with satisfactory results. I would not recommend its use in infected cases, because the first consideration must be thorough drainage and good antisepsis; neither would I make a bone flap or expose the bone chips in cases where the scalp is in fected, as it was in my case. I thank you for the free discus sion of my paper.

Acne Rosacea.-Jadassohn applies the following salve with massage at night, after washing the face in hot water with soap or borax if there is much greasy secretion: Ichthyol 1 to $5 \mathrm{gm}$., resorcin 1 to $3 \mathrm{gm}$. adip. lan. $25 \mathrm{gm}$., ol. oliv. $10 \mathrm{gm}$., aq. ad. $50 \mathrm{gm}$. Wash off in the morning with hot water and afterward with spirit. $-N$. Y. Med. Woch., January.

\section{THE ACCOMMODATION}

Read before the Chicago Academy of Medicine, Jan. 13, 1899 BY J. G. HUIZINGA, M.D.

PROFESSOR OF OPHTHALMOLOGY, CHICAGO EXE, EAR, NOSE AND THROAT COLLEGE; OPHTHALMOLOGIST TO HALSTED STREET DISPENSARY. chicago.

Accommodation is that function of the visual organ whereby it is able to adjust itself so that rays of light coming from different distances may all be focused upon the retina consecutively. In other words, it is that power of the eye whereby the refraction of the organ may be increased or decreased within certain limits at will.

"The contraction of the ciliary muscles causes the zone of Zinn to advance, and thus diminishes the traction exerted by the latter upon the crystalline. The lens, abandoned to itself, assumes the form which the elasticity of its fibers naturally gives it, and becomes more convex, especially its anterior surface.

"When the innervation ceases, the ciliary muscles are relaxed; the ciliary processes become tense, and stretch the zone of Zinn, which, in turn, flattens the crystalline by exerting upon it a traction in the direction of its equator." Thus during a large portion of our lives the lens is compressed and cramped into a shape that is not natural to it. The result is that the lens ultimately assumes that shape which it would possess when the zonula is in a state of maximum tension. Any relaxation of the zonula at this time will have no effect upon the form of the lens because the lens fibers have gradually accommodated themselves to the conditions imposed upon them.

Another result of this tension of the zonula is that the lens " changes in its constitution." The peripheral layers of the crystalline being compressed and more dense increases their index of refraction, so that in the latter years of life, the index of refraction of the nucleus and the peripheral layer is very nearly alike, and the lens becomes more ana more homogeneous as age advances.

The difference between the refraction of the lens with the zonula tense and its refraction with the zonula relaxed is the measure of the range of accommodation. The normal range of accommodation is a very vague term, and may signify almost anything. What is normal in one case may be abnormal in another, and vice versa. But while this may vary considerably in different individuals and at different ages, there ought nevertheless to be, what, for want of a better term, we may call, a proper range of accommodation, and where this is deficient it must be supplied by artificial means, i.e., convex lenses.

By the proper range of accommodation we mean that condition of ciliary muscles and lens in which the action of the former combined with the inherent elasticity of the latter is sufficient to change the refraction of the eye to such a degree as is necessary to meet all the requirements of vision in the discharge of the particular duties of the patient without requiring the muscle to expend more than two-thirds or three-fourths of its strength and endurance. It is possible for a patient to have a normal without a proper range of accommodation. For instance: a man 29 years old whose normal range of accommodation is equal to 7 diopters, but who is hyperopic to the extent of 3 diopters, and who, in the discharge of his ordinary duties, requires 4 diopters of accommodation, is obliged to use constantly his entire accommodation, consequently, the ciliary muscle must con- 